\title{
SOME CONJECTURES ON ELLIPTIC CURVES OVER CYCLOTOMIC FIELDS
}

BY

D. GOLDFELD AND C. VIOLA

\begin{abstract}
We give conjectures for the mean values of Hasse-Weil type $L$-functions over cyclotomic fields. In view of the Birch-Swinnerton-Dyer conjectures, this translates to interesting arithmetic information.
\end{abstract}

1. In $1967 \mathrm{~A}$. Weil [6] showed that if the Dirichlet series

$$
L_{1}(s)=\sum_{n=1}^{\infty} a(n) n^{-s}, \quad L_{1}(s, \chi)=\sum_{n=1}^{\infty} a(n) \chi(n) n^{-s} \quad(\chi \bmod q)
$$

satisfy the functional equations

$$
\begin{aligned}
& (\sqrt{N} / 2 \pi)^{s} \Gamma(s) L_{1}(s)=w_{1}(\sqrt{N} / 2 \pi)^{k-s} \Gamma(k-s) L_{1}(k-s), \quad\left|w_{1}\right|=1, \\
& (\sqrt{N} / 2 \pi q)^{s} \Gamma(s) L_{1}(s, \chi)=w_{\chi}(\sqrt{N} / 2 \pi q)^{k-s} \Gamma(k-s) L_{1}(k-s, \bar{\chi}),
\end{aligned}
$$

$$
w_{\chi}=w_{1} \varepsilon(q) \frac{\tau_{\chi}}{\tau_{\bar{\chi}}} \chi(-N), \quad \tau_{\chi}=\sum_{a=1}^{q} \chi(a) e^{2 \pi i a / q},
$$

for "sufficiently many" $q$ such that $(q, N)=1$ and all primitive characters $\chi \bmod q$, where $N$ and $k$ are positive integers and $\varepsilon$ is a primitive character $\bmod N$, then $f(z)=$ $\sum_{n=1}^{\infty} a(n) e^{2 \pi i n z}$ is a modular form with multiplier $\varepsilon$ of weight $k$ for the congruence subgroup

$$
\Gamma_{0}(N)=\left\{\left(\begin{array}{ll}
a & b \\
c & d
\end{array}\right) \mid a, b, c, d \in \mathbf{Z}, a d-b c=1, c \equiv 0(\bmod N)\right\} .
$$

Our aim is to propose some conjectures on the asymptotic behaviour of the mean value

$$
S(X ; h)=\sum_{\substack{p \leq X \\ p \equiv \equiv(h)}} \prod_{\substack{x_{\bmod } p \\ \chi^{h}=\chi_{0} \\ r \neq \neq \chi_{0} \text { for } r<h}} L_{1}(k / 2, \chi) \quad(h \geqq 2 \text { a fixed integer })
$$

where the sum is restricted to primes $p$, and $\chi_{0}$ is the principal character $\bmod p$.

Let $L_{h}(s, \chi)=\sum_{n=1}^{\infty} a\left(n^{h}\right) \chi(n) n^{-s}$. If $L_{1}(s)$ satisfies (1) and (2), then we propose the following

Received by the editors June 12, 1981.

1980 Mathematics Subject Classification. Primary 12A70.

(1) 1983 American Mathematical Society 0002-9947/81/0000-0759/\$09.00 
Main Conjecture. For $h=2\left(\chi^{2}=\chi_{0}, \chi \neq \chi_{0}, \chi \bmod p\right)$ :

$$
\begin{aligned}
S(X ; 2) & =\sum_{p \leq X} L_{1}(k / 2, \chi) \\
& \sim \sum_{p \leq X} \frac{1+w_{1} \varepsilon(p) \chi(-N)}{\Gamma(k / 2)} \cdot \frac{1}{2 \pi i} \int_{c-i \infty}^{c+i \infty} \Gamma(k / 2+z)\left(\frac{\sqrt{N}}{2 \pi} p\right)^{2} L_{2}\left(k+2 z, \chi_{0}\right) \frac{d z}{z} .
\end{aligned}
$$

For $h>2$ :

$$
\begin{aligned}
S(X ; h) \sim & \sum_{\substack{p \leq X \\
p \equiv 1(h)}} \frac{1+\left(w_{1} \varepsilon(p)\right)^{\phi(h)}}{\Gamma(k / 2)^{\phi(h)}} \\
& \cdot \frac{1}{2 \pi i} \int_{c-i \infty}^{c+i \infty} \Gamma(k / 2+z)^{\phi(h)}\left(\frac{\sqrt{N}}{2 \pi} p\right)^{\phi(h) z} H(z)^{\phi(h) / 2} \frac{d z}{z} \quad(c>0),
\end{aligned}
$$

where

$$
H(z) \equiv \sum_{n=1}^{\infty} \frac{a(n)^{2} \chi_{0}(n)}{n^{k+2 z}}+L_{h}\left(h(k / 2+z), \chi_{0}\right)^{2}
$$

The integrals in the above conjecture can be easily evaluated asymptotically by shifting the line of integration and computing the residues at $z=0$. If we assume that $L_{1}(s)$ has an Euler product of the form

$$
L_{1}(s)=\prod_{p \mid N}\left(1-\frac{a(p)}{p^{s}}\right)^{-1} \cdot \prod_{p \nmid N}\left(1-\frac{\gamma_{p}}{p^{s}}\right)^{-1}\left(1-\frac{\bar{\gamma}_{p}}{p^{s}}\right)^{-1}
$$

with $\left|\gamma_{p}\right|^{2}=p^{k-1}$ (see [1]), then we have the following

Proposition. Assuming the Main Conjecture and the Euler product (3), it follows that

$$
S(X ; 2) \sim \frac{48 \pi}{N} \prod_{p \mid N}\left(1-p^{-2}\right)^{-1} \cdot\langle f, f\rangle \cdot \frac{X}{\log X}
$$

where

$$
\langle f, f\rangle=\int_{\Gamma_{0}(N) \backslash H}\left|\sum_{n=1}^{\infty} a(n) e^{2 \pi i n z}\right|^{2} y^{k-2} d x d y
$$

is the Petersson inner product of $f$ with itself;

(ii) For $h>2$,

$$
S(X ; h) \sim 2\left(\frac{24 \pi}{N} \prod_{p \mid N}\left(1+p^{-1}\right)^{-1} \cdot\langle f, f\rangle\right)^{\phi(h) / 2} \frac{X(\phi(h) \log X)^{\phi(h) / 2-1}}{\left(\frac{1}{2} \phi(h)\right) !} .
$$

In the case that the weight $k$ is 2 , that (1), (2) and (3) are satisfied and the $a(n)$ are rational, it is known (see [4, Theorems 7.14, 7.15]) that $L_{1}(s)$ is the Hasse-Weil $L$-function of some elliptic curve $E$, and $\langle f, f\rangle$ can always be expressed as the product of an algebraic number, a power of $\pi$, and the two periods of $E$. For example (see [5]), when $k=2, N=11$ there is a unique cusp form of weight 2:

$$
f(z)=e^{2 \pi i z} \prod_{n=1}^{\infty}\left(1-e^{2 \pi i n z}\right)^{2} \prod_{n=1}^{\infty}\left(1-e^{2 \pi i n z}\right)^{2}=\sum_{n=1}^{\infty} a(n) e^{2 \pi i n z},
$$


and $L_{1}(s)=L_{E}(s)=\sum_{n=1}^{\infty} a(n) n^{-s}$ is the Hasse-Weil $L$-function of the elliptic curve $E: y^{2}+y=x^{3}-x^{2}$. For this curve, our conjectures take the following form:

$$
\begin{aligned}
& S(X ; 2) \sim \frac{11}{5 \pi} \Omega^{+} \Omega^{-} \frac{X}{\log X}, \\
& S(X ; h) \sim 2\left(\frac{\Omega^{+} \Omega^{-}}{\pi}\right)^{\phi(h) / 2} \frac{X(\phi(h) \log X)^{\phi(h) / 2-1}}{\left(\frac{1}{2} \phi(h)\right) !} \quad(h>2) .
\end{aligned}
$$

Here $\Omega^{+}=0.6346047 \cdots$ and $\Omega^{-}=1.4588166 \cdots$ are the real period and the absolute value of the imaginary period of $E$, respectively.

Now let $p$ be an odd prime. The Hasse-Weil $L$-function of $E$ over the cyclotomic field $\mathbf{Q}(\sqrt[p]{1})$ is given by

$$
L_{\mathbf{Q}(\mathbb{R} \mathcal{T}) \backslash E}(s)=\prod_{\chi \bmod p} L_{E}(s, \chi) .
$$

It is reasonable to expect that the average value (as $p$ varies) is given by

average value of $L_{\mathbf{Q}(\ell T) \backslash E}(1) \sim L_{E}(1) \cdot \frac{11}{5 \pi} \Omega^{+} \Omega^{-} \cdot \prod_{\substack{h(p-1) \\ h>2}} \frac{2\left(\frac{\phi(h)}{\pi} \Omega^{+} \Omega^{-} \log p\right)^{\phi(h) / 2}}{\phi(h)\left(\frac{1}{2} \phi(h)\right) !}$.

Sharper forms of part (ii) of the Proposition can be derived on assuming the analytic continuation of $L_{h}(s)$ for $h>2$. Then there will be extra terms involving lower powers of $\log X$, whose coefficients are expressible in terms of the special values of $L_{h}(s)$, some of which can be given by the conjectures of Deligne [2].

2. In order to lend credence to our Main Conjecture, we give the following arguments. Firstly, the conjecture for $S(X ; 2)$ has already been dealt with in [3]. We therefore consider $S(X ; h)$ for $h>2$. For any prime $p \equiv 1(\bmod h)$ there are $\phi(h)$ characters $\bmod p$ of exact order $h$, and moreover these characters are all primitive. It follows by (2) that, for $p \nmid N$,

$$
\begin{gathered}
\left(\left(\frac{\sqrt{N}}{2 \pi} p\right)^{s} \Gamma(s)\right)^{\phi(h)} \prod_{\chi \bmod p}^{\prime} L_{1}(s, \chi)=W\left(\left(\frac{\sqrt{N}}{2 \pi} p\right)^{k-s} \Gamma(k-s)\right)^{\phi(h)} \prod_{\chi \bmod p}^{\prime} L_{1}(k-s, \chi), \\
W=W(p, h)=\prod_{\chi \bmod p}^{\prime} w_{\chi}=\left(w_{1} \varepsilon(p)\right)^{\phi(h)},
\end{gathered}
$$

where $\Pi^{\prime}$ means that the product is taken over all characters of exact order $h$, which we denote by $\chi_{1}, \ldots, \chi_{\phi(h)}$. By Lavrik's method (see [3]), we have

$$
\begin{aligned}
\prod^{\prime} L_{1}(k / 2, \chi)=\frac{1+W}{\Gamma(k / 2)^{\phi(h)}} \sum_{n_{1}} \cdots \sum_{n_{\phi(h)}} a\left(n_{1}\right) & \cdots a\left(n_{\phi(h)}\right) \chi_{1}\left(n_{1}\right) \\
\cdots & \chi_{\phi(h)}\left(n_{\phi(h)}\right)\left(n_{1} \cdots n_{\phi(h)}\right)^{-k / 2}
\end{aligned}
$$

$$
\cdot \frac{1}{2 \pi i} \int_{c-i \infty}^{c+i \infty}\left(\Gamma\left(\frac{k}{2}+z\right)\left(\frac{\sqrt{N}}{2 \pi} p\right)^{z}\right)^{\phi(h)}\left(n_{1} \cdots n_{\phi(h)}\right)^{-z} \frac{d z}{z} .
$$

Now, summing over $p$ should give a lot of cancellation except for those $\phi(h)$-tuples 
$\left(n_{1}, \ldots, n_{\phi(h)}\right)$ for which

$$
\prod_{i=1}^{\phi(h)} \chi_{i}\left(n_{i}\right)=1
$$

for all $p \nmid n_{i}(i=1, \ldots, \phi(h))$. We can arrange the characters so that $\chi_{i}=\bar{\chi}_{\phi(h)-i+1}$. It follows that

$$
\chi_{i}\left(n_{i}\right) \chi_{\phi(h)-i+1}\left(n_{\phi(h)-i+1}\right)=1
$$

whenever $n_{i}=n_{\phi(h)-i+1}$ and $p \nmid n_{i}$. Also $\chi_{i}\left(n_{i}\right)=1$ whenever $n_{i}=m_{i}^{h}$ is an $h$ th-power and $p \nmid m_{i}$. Combinations of these two cases are the only ways in which the aforementioned tuples can be constructed. Hence, every tuple $\left(n_{1}, \ldots, n_{\phi(h)}\right)$ satisfying (5) is given as follows. Let $0 \leqq r \leqq \frac{1}{2} \phi(h)$; choose an $r$-tuple $\left(i_{1}, \ldots, i_{r}\right)$ with $1 \leqq i_{1}<\ldots$ $<i_{r} \leqq \frac{1}{2} \phi(h)$. Put $n_{i j}=n_{\phi(h)-i j+1}(j=1, \ldots, r)$. Also let $n_{i}=m_{i}^{h}$ be a perfect $h$ th-power for $i \neq i_{j}, i \neq \phi(h)-i_{j}+1(j=1, \ldots, r)$. Since there are exactly $(\underset{r}{\phi(h) / 2})$ such $r$-tuples, it is reasonable to expect that, after summing (4) over primes $p \equiv 1(\bmod h), S(X ; h)$ should be given asymptotically as

$$
\begin{aligned}
S(X ; h) \sim & \sum_{\substack{p \leq X \\
p=1(h)}} \frac{1+W}{\Gamma(k / 2)^{\phi(h)}} \sum_{r=0}^{\phi(h) / 2}\left(\begin{array}{c}
\frac{1}{2}(\phi h) \\
r
\end{array}\right) \\
& \times \frac{1}{2 \pi i} \int_{c-i \infty}^{c+i \infty}\left(\Gamma\left(\frac{k}{2}+z\right)\left(\frac{\sqrt{N}}{2 \pi} p\right)^{z}\right)^{\phi(h)} \\
& \cdot\left(\sum_{n=1}^{\infty} \frac{a(n)^{2} \chi_{0}(n)}{n^{k+2 z}}\right)^{r}\left(\sum_{m=1}^{\infty} \frac{a\left(m^{h}\right) \chi_{0}(m)}{m^{h(k / 2+z)}}\right)^{\phi(h)-2 r} \frac{d z}{z} \\
= & \sum_{\substack{p \leq X \\
p \equiv 1(h)}} \frac{1+W}{\Gamma(k / 2)^{\phi(h)}} \cdot \frac{1}{2 \pi i} \int_{c-i \infty}^{c+i \infty}\left(\Gamma\left(\frac{k}{2}+z\right)\left(\frac{\sqrt{N}}{2 \pi} p\right)^{z}\right)^{\phi(h)} H(z)^{\phi(h) / 2} \frac{d z}{z} .
\end{aligned}
$$

In order to derive the Proposition from the Main Conjecture, note that the RankinSelberg $L$-function $\sum_{n=1}^{\infty}|a(n)|^{2} n^{-s}$ has a simple pole at $s=k$ with residue

$$
\frac{48 \pi}{N} \prod_{p \mid N}\left(1+p^{-1}\right)^{-1} \cdot\langle f, f\rangle .
$$

If $L_{1}(s)$ satisfies (3), then the coefficients $a(n)$ are real, and $w_{1} \varepsilon(p)= \pm 1$. Also, $L_{h}(s)$ is regular for $\operatorname{Re}(s)>1+h(k-1) / 2$. Hence, for $h>2, H(z)$ is regular for $\operatorname{Re}(z)>$ $\frac{1}{h}-\frac{1}{2}$ except for a simple pole at $z=0$, with residue

$$
\frac{24 \pi}{N} \prod_{p \mid N}\left(1+p^{-1}\right)^{-1} \cdot\langle f, f\rangle \text {. }
$$

Shifting the line of integration to $c=\frac{1}{h}-\frac{1}{2}+\varepsilon\left(0<\varepsilon<\frac{1}{2}-\frac{1}{h}\right)$ and using standard estimates for the growth of the Rankin-Selberg $L$-function, the proposition follows on computing the main term of the residue and applying the prime number theorem for arithmetic progressions.

\section{REFERENCES}

1. P. Deligne, La conjecture de Weil. I, Inst. Hautes Études Sci. Publ. Math. 43 (1973), 273-307.

2. - Valeurs de fonctions L et périodes d'intégrales, Proc. Sympos. Pure Math., vol. 33, part 2, Amer. Math. Soc., Providence, R.I., 1979, pp. 313-346. 
3. D. Goldfeld and C. Viola, Mean values of L-functions associated to elliptic, Fermat and other curves at the centre of the critical strip, J. Number Theory 11 (1979), 305-320.

4. G. Shimura, Introduction to the arithmetic theory of automorphic functions, Princeton Univ. Press, Princeton, N.J., 1971.

5. J. Tate, The arithmetic of elliptic curves, Invent. Math. 23 (1974), 179-206.

6. A. Weil, Über die Bestimmung Dirichletscher Reihen durch Funktionalgleichungen, Math. Ann. 168 (1967), 149-156.

Department of Mathematics, Massachusetts Institute of Technology, Cambridge, MasSACHUSETTS 02139

Istituto di Matematica, Università di Pisa, Pisa, Italy 